\title{
Alzheimer's Disease Genetics
}

\author{
Matthew Schreiber • Thomas D. Bird • Debby W. Tsuang
}

Published online: 4 October 2014

(C) Springer International Publishing AG (outside the USA) 2014

\begin{abstract}
Alzheimer's disease (AD) is the leading cause of dementia, and each year the number of individuals suffering from its consequences continues to increase, yet current treatments only delay $\mathrm{AD}$ symptom progression and do not modify the underlying causes of the disease. Recently, the genetics of AD have been explored with increasing scope and intensity, revealing that while only a portion of $\mathrm{AD}$ is familial, genetics play a strong role even in the common, apparently sporadic cases of AD. Initially, autosomal dominant forms of AD were discovered in genes that are now considered central to the pathogenesis of the disease. Then, in the next phase of genetic discovery, apolipoprotein $\mathrm{E}(A P O E) \varepsilon 4$ alleles were very strongly associated with $\mathrm{AD}$ risk; these findings and their role in the pathobiology of $\mathrm{AD}$ have been reviewed extensively elsewhere (Bettens et al. Lancet Neurol. 12(1):92-104, 2013). This current review discusses recent genetic directions in $\mathrm{AD}$ research and updates an earlier review, which detailed the biology and fundamental genetic findings in $\mathrm{AD}$ up to about 2009 (Bekris et al. J Geriatr Psychiatry Neurol. 23(4):213-27, 2010), by focusing on new findings in the rapidly changing landscape of $\mathrm{AD}$ genetic research and demonstrating that multiple complementary genetic approaches, particularly in
\end{abstract}

\footnotetext{
M. Schreiber

Mental Health Service, VA Puget Sound Health Care System, Seattle, WA, USA

M. Schreiber $\cdot$ D. W. Tsuang $(\bowtie)$

Department of Psychiatry and Behavioral Sciences, University of Washington, Seattle, WA, USA

e-mail: dwt1@uw.edu

T. D. Bird $\cdot$ D. W. Tsuang

Geriatric Research Education Clinical Center, VA Puget Sound

Health Care System, Seattle, WA, USA

T. D. Bird

Department of Neurology, University of Washington, Seattle, WA, USA
}

large-scale genomic-level investigations, are leading to rapid and accelerating progress.

Keywords Alzheimer's disease · Genetics · GWAS · Next-generation sequencing

\section{Introduction: Recent Perspective on Alzheimer's disease (AD) Genetics}

Alzheimer's disease (AD) is often considered from distinct genetic perspectives [1] and grouped into early-onset $\mathrm{AD}$ (EOAD) (i.e., age at onset $<65$ years) and late-onset $\mathrm{AD}$ (LOAD). At the same time, $\mathrm{AD}$ is often viewed as familial (i.e., when two or more family members are affected) or sporadic. However, even in sporadic AD, it is expected that genetics, interacting with environment, are strongly involved. Recent genetic findings in AD are built upon earlier discoveries that revealed the autosomal dominant forms of $\mathrm{AD}$ [related to mutations in $A P P$ (amyloid precursor protein) and PSEN1 and PSEN2 (presenilin-1 and -2)]. These autosomal dominant forms of $\mathrm{AD}$ are most frequently found in $\mathrm{EOAD}$ and account for about $2 \%$ of AD cases overall [1]. Despite the tantalizing indication that a substantial amount of the genetic contribution to $\mathrm{AD}$ remained following the discovery of the autosomal dominant forms of $\mathrm{AD}$ and the apolipoprotein $\mathrm{E}$ $(A P O E)$ genotype risk factor, which can be seen as acting as a semi-dominant, incompletely penetrant genetic cause of $\mathrm{AD}$, it is only recently that more advanced genetic analyses have begun uncovering additional genetic risk factors for $\mathrm{AD}$. These new methods fall into two general categories: genome-wide association studies (GWASs) for the detection of common alleles and, more recently, studies using wholegenome and whole-exome sequencing (WGS and WES, respectively) for the detection of rare alleles. We review findings from these types of studies in $\mathrm{AD}$ in this article. 


\section{A Multitude of Risk Factors: The Genome-Wide Association Study (GWAS) Harvest}

\section{GWASs Based on Disease Phenotype: Large-Scale Studies}

The first wave of GWASs in AD focused on LOAD. Candidate loci with genome-wide significant statistical associations with $\mathrm{AD}$ were identified, but these findings proved difficult to replicate, and few, if any, of these initial genetic associations have remained. However, this first wave of studies enabled researchers to strengthen their approaches in GWAS design and execution, efforts that have borne fruit in the second wave of GWASs, which we examine here. In particular, larger sample sizes, the growing sophistication of statistical methods, especially those related to the pooling of data in meta-analyses, and the use of multi-staged studies that include both discovery and confirmation stages have together greatly increased the power to detect relatively small genetic influences on $\mathrm{AD}$. The limitations of GWASs are well-known, including, most prominently, the fact that the findings are often hard to decipher: significant markers from GWASs do not always implicate specific genes, and the relationships between implicated single nucleotide polymorphisms (SNPs) and relevant genes are often unclear. However, because GWASs point strongly toward genes that neighbor or incorporate the identified SNPs, researchers have nonetheless been able to identify an increasing number of specific genes that are now thought to increase the risk for AD. It should be noted that additional levels of complexity have also been incorporated into such analyses, including replications across different populations, examinations of the impact of allele frequencies in these different populations, and methods that control for potentially confounding factors. For example, in a study combining a GWAS with a family-study structure [2] and a methodology that carefully controlled for $A P O E$ allele status, some previously significant genetic variants appeared to be weakened by controlling for $A P O E$ while others were confirmed.

The second wave of GWASs have identified a large group of genetic risk factors. Those with the strongest evidence include $C L U$ (clusterin), CRI (complement receptor 1), and PICALM (phosphatidylinositol-binding clathrin assembly protein), as discussed below. Others include BIN1 (bridging integrator protein 1), EPHA1 (ephrin receptor A1), $A B C A 7$ (adenosine triphosphate-binding cassette protein A7), the MS4A (membrane-spanning A4) genes, CD33 (sialic acidbinding immunoglobulin-like lectin 33), and CD2AP (CD2associated protein). Perhaps most intriguingly, these findings are beginning to suggest a pattern of non-random association with biochemical functional groups of genes, and, ultimately, this pattern is likely to be where GWASs will make their strongest contribution. One suggested grouping [3] of these GWAS-based candidates points to increasingly strong associations with $\beta$-amyloid, lipid export, immune, and synaptic function pathways; these authors note that some genes span and interconnect these pathways, which strengthens the case that the pathways are involved in AD pathobiology.

In fact, prior to about 2009, apart from the clearly autosomal dominant forms of $\mathrm{AD}$, only $A P O E$ was found, robustly and repeatedly, to be a genetic risk factor for AD. The difficulty with the first wave of GWAS replication pointed to a need for larger sample sizes and for the more robust application of meta-analytic techniques, as well as increasingly wide networks of consortia to undertake these studies. Since then, consortia have been pooling together large GWAS datasets in meta-analysis-like approaches. With this unprecedented power, a growing list of markers that are thought to be associated with specific genes and candidate risk factor genes has emerged, and some of these risk factors have been replicated. Note that these genes are not necessarily considered causative; rather, they are relatively common alleles that either increase or decrease $\mathrm{AD}$ risk. Each individual genomic location may contribute a small amount of risk. Perhaps most significantly, these genes are painting an ever more detailed picture of the pathways involved in $\mathrm{AD}$ pathogenesis. Ultimately, an understanding of these genes will greatly enhance our ability to develop specific, disease-modifying treatments that have been lacking to this point and that are critically needed.

A second-wave GWAS that included a two-stage design and more than 16,000 individuals [4] was the first to reliably identify genetic contributors beyond $A P O E$. In this study, $C L U$ and PICALM surpassed genome-wide statistical significance, while BIN1 reached "suggestive" statistical significance, and, as expected, $A P O E$ was clearly associated with $\mathrm{AD}$ risk. A later study then replicated $C L U$ and PICALM but found that these loci did not add to a predictive model that included the $A P O E$ genotype. However, these authors note that this does not preclude a biochemically important role for $C L U$ and PICALM in AD [5]. The study also found that two other markers, near BIN1 and MARK4 [microtubule-associated protein (MAP)/microtubule affinity-regulating kinase 4], reached significance. Another large GWAS (2,032 cases) that was conducted early in the second wave used a multi-stage analysis that provided for within-study replicative evidence to report significant risk effects linked to $C L U$, again, and $C R 1$, both with increased risk odds ratios (ORs) of around 1.22 [6]. The authors of this study note that both $C L U$ and $C R 1$ had been previously linked to $\mathrm{AD}$ pathobiology, in particular with the clearance of $\beta$-amyloid.

The Alzheimer's Disease Genetics Consortium (ADGC) carried out a large US GWAS with a discovery stage, two replication stages, and a meta-analysis [7]. In this study, loci associated with MS4A4A, CD2AP, EPHA1, and CD33 were shown to significantly alter the risk of LOAD. In addition, CR1, CLU, BIN1, and PICALM findings were replicated, but findings related to EXOC3L2 (exocyst complex component 3- 
like 2) were not replicated. The replicated genes were estimated to have population-attributable factors between 2.72 and 5.97 \%. In a companion European Genetic and Environmental Risk for Alzheimer's Disease Consortium (GERAD) GWAS that included a meta-analysis, $M S 4 A 4 A, E P H A 1, C D 2 A P$, and $C D 33$ were also identified, and a marker at $A B C A 7$ also reached significance [8]. This study also replicated evidence for an association between $\mathrm{AD}$ and BIN1 and CR1.

Most recently, in the largest GWAS published to date from the International Genomics of Alzheimer's Project (IGAP), which included 74,046 subjects and a large, two-stage metaanalysis, 19 loci reached significance [9]; 11 are described as novel, while 8 provided replicative evidence. The previously known loci were related to $C R 1, B I N 1, C D 2 A P, E P H A 1$, $C L U, M S 4 A, P I C A L M, A B C A 7$, and CD33. Among the novel loci associated with AD risk in this study, the authors particularly highlighted markers near HLA-DRB (human leukocyte antigen-DRB) (encoding major histocompatibility complex class II, DR $\beta \mathrm{B}$ ) and ZCWPWI (zing finger, CW type, with PWWP domain). Evidence was also found tying SORL1 (sortilin-related receptor L) to AD risk; this is of interest because this locus has been identified previously in other studies. These findings illustrate the way that evidence for specific genes can accumulate when multiple approaches are leveraged.

At the same time, complementary evidence has emerged from GWASs of a relatively smaller scale that in an effort to assess the commonality of genetic influences on AD risk has focused on more specific ethnic groups. A two-stage GWAS in 1,009 African Americans with LOAD, for example, identified $A P O E$ as well as confirmatory evidence of an association between LOAD and $A B C A 7$ [10]. The study, which included a second stage for replication and comparison, also identified CLU, PICALM, BIN1, EPHA1, MS4A, and CD33 as significant, though, interestingly, the related risk was not always in the same direction as in previous studies; the authors suggest that while the same genes may contribute to $\mathrm{AD}$ pathogenesis in African Americans, the causal variants may not be identical. This study also found evidence that suggests PROX1 (prospero homeobox protein 1) and CNTNAP2 (contactin-associated protein-like 2) as additional candidate genes for AD risk.

Alternatively, smaller GWASs can be successful by employing novel or variant analysis methods and approaches. A recent GWAS aimed to reveal patterns of associations within genes by employing a "mega meta-analysis" and an alternative gene-wide analysis; this study led to confirmatory evidence for 20 genes previously identified in second-wave GWASs and also identified two novel loci, TP53INP1 (tumor protein p53-inducible nuclear protein 1) and IGHV1-67 (IGHV1-67 immunoglobulin heavy variable 1-67, pseudogene) [11]. Likewise, a similar study implicated three genes near recently reported SNPs [ZCWPW1, NDUFS3 (nicotinamide adenine dinucleotide [NADH] dehydrogenase [ubiquinone] Fe-S protein 3), and MTCH2 (mitochondrial carrier 2)] [9]. More modestly scaled studies have also been valuable in that they have allowed confirmatory evidence to emerge regarding the markers uncovered in larger studies. A study of 1,291 novel cases with LOAD that included a second meta-analysis step with a larger group, for example, reconfirmed six previously identified markers (inclusive of APOE) [12]: PICALM, BIN1, ABCA7, MS4A4/MS4A6E, and EPHA1. Additionally, a suggestive novel marker was identified as potentially significant in the $P P P 1 R 3 B$ (protein phosphatase 1, regulatory subunit $3 \mathrm{~B}$ ) gene. Ideally, such smaller studies serve to reinforce and complement large-scale studies and to enrich the gene discovery process.

\section{Endophenotype-Based GWASs}

Along with conventional GWASs that are based on the clinical diagnosis of $A D$, recent GWASs have utilized endophenotypes. These endophenotype-based studies allow investigators to explore different neurobiological aspects of $\mathrm{AD}$, potentially revealing information that is not accessible when studying $\mathrm{AD}$ as the only phenotype. However, because these studies require a more detailed analysis of individual cases beyond the binary presence or absence of the $\mathrm{AD}$ diagnosis, they have not yet been scaled up to the large study sizes that are more typical of the second wave of GWASs. Still, although the small sample sizes reduce the power of these studies to detect the relatively small genetic effects expected, interesting and novel findings have emerged.

Neuritic Plaque Burden In a relatively small sample for which detailed pathological data had been obtained, a candidatebased GWAS approach was undertaken to explore genetic links to neuritic plaque burden [13]. In the GWAS phase of the study, no genome-wide significant SNPs were identified. However, a suggestive link was made to novel candidate genes, including KCNIP4 ( $\mathrm{Kv}$ channel interacting protein 4), PTGS1 (prostaglandin-endoperoxide synthase 1), and the HLA locus. The latter is particularly interesting given the possible links between immune function and $\mathrm{AD}$. In the candidate gene analyses, $A P O E, C R 1, A B C A 7$, and $C D 2 A P$ were found to be linked to neuritic plaque burden as well, thereby connecting findings from previously conducted disease-based, large-scale GWASs to an endophenotype.

Rate of Cognitive Decline A recent two-stage GWAS used a functional marker of disease progression to find genes related to the rate of cognitive decline in $\mathrm{AD}$ [14]. Because there is very little known about the variability in the rate of the progression of $\mathrm{AD}$, this study potentially represents a highly fruitful area for research. Although the sample size of the GWAS was small (303 cases), SPON1 (spondin 1) was 
identified in both the discovery and replication phases of the study; its minor allele was linked to slowed disease progression. The authors also linked a novel genetic variant to the progression of $\mathrm{AD}$ that had not been found in larger studies that used clinical $\mathrm{AD}$ as the phenotype.

Cerebrospinal Fluid Tau and p-Tau Levels A GWAS that was conducted in 1,269 AD cases with cerebrospinal fluid (CSF) tau and p-tau levels as biomarkers revealed four loci that were significant across the genome [15]: $A P O E$, and markers at $3 \mathrm{q} 28$ (which is not clearly linked to any gene), GLIS3 (GLIS family zinc finger 3), and 6p21.1 in the TREM (triggering receptor expressed on myeloid cells) gene cluster. These four loci accounted for $22 \%$ of the genetic variation in CSF p-tau levels. However, not all of these markers were linked to AD risk itself.

Plasma Amyloid Peptide Levels A GWAS was conducted in a sample of older European adults with plasma amyloid peptide levels used as an $\mathrm{AD}$ endophenotype [16]. Although no markers reached genome-wide significance in this study, there were suggestive links to several interesting markers.

Taken together, these studies, though as yet small, already suggest that pursuing GWASs using endophenotypes, whether as biomarkers or functional measures, can yield novel genetic associations that are not identified in larger, more typical GWASs. Even with appropriate caution, given that these study findings are not as yet replicated (and keeping in mind the negative fate of findings from the first wave of GWASs), these endophenotype studies are a promising step toward uncovering functionally relevant genetic contributors to $\mathrm{AD}$ pathogenesis.

\section{Whole-Genome and Whole-Exome Sequencing}

Our ability to sequence whole exomes and whole genomes is progressing rapidly and is just beginning to be applied to the exploration of the genetics of AD. For example, in a familial $\mathrm{AD}$ dataset that excluded the three major autosomal forms of $\mathrm{AD}$, exome sequencing identified rare variants in SORL1, a gene that had been previously identified in other settings as playing a role in $\beta$-amyloid production [17]. In this case, these rare SORL1 variants would not have been associated with $\mathrm{AD}$ in standard GWASs, but they were identified from exome sequencing in a group of families known to be at high risk for $\mathrm{AD}$ due to their family history.

TREM2 Jonsson et al. used extensive WGS data in a set of 2,261 Icelanders to identify variants in TREM2. Their genetic analyses found a rare missense mutation in TREM2 (predicted mutation $\mathrm{R} 47 \mathrm{H}$ ) that was associated with the risk of $\mathrm{AD}$ with an OR estimated at 2.09-4.09 (95\% confidence interval), and they replicated this finding in other datasets [18]. A combined genome, exome, and Sanger sequencing approach was used to independently identify the same mutation, associating this heterozygous rare variant in TREM2 with an increased risk of LOAD; a brief letter describing a meta-analysis also reported this finding, with a similar risk (OR 2.65-4.35) [19]. TREM2 had previously been identified as harboring the causative mutation in autosomal recessive Nasu-Hakola disease (polycystic lipomembranous osteodysplasia with sclerosing leukoenchepalopathy), a disease that is distinct from $\mathrm{AD}$, yet, interestingly, one individual with Nasu-Hakola disease had been identified as bearing senile plaques and neurofibrillary tangles long before the genetic source of the disease was known [20]. As with hits from GWASs, these exome sequencing findings suggest clues toward identifying the functional pathways in $\mathrm{AD}$, as TREM2 has been implicated in immune function in the brain. Recently, a TREM-like 2 coding missense variant was identified as potentially playing a protective role in $\mathrm{AD}$, further suggesting that this family of receptors may play crucial roles in $\mathrm{AD}$ pathogenesis [21].

PLD3 WES can be particularly effective at revealing lowfrequency coding variations with relatively large effects on AD risk. PLD3 (phospholipase D family, member 3) has been identified using a WES approach in 14 large LOAD families [22]. Unlike GWAS methods, this approach by definition directly identifies the mutations linked to risk. In this case, $P L D 3$ is an especially intriguing finding, as it has been linked to $A P P$ processing, and the risk associated with these variants is up to twofold.

$A P P$ A major familial form of EOAD is known to be caused by $A P P$ mutations, but recently an allele of $A P P$ was found to be protective. In a study interrogating large Icelandic WGS data [23], a predicted A673T change in $A P P$ reduced both the risk of $\mathrm{AD}$ and of cognitive decline in older subjects without $\mathrm{AD}$. This mutation may function by reducing beta cleavage in APP, as in vivo studies revealed a $40 \%$ decline in amyoidogenic peptides. From a genetic perspective, this study illustrates how WGS can lead to further studies that uncover surprising new genetic influences on AD.

NOTCH3 Albeit at a much smaller scale than the studies of APP, a study utilized WES to identify an AD-related mutation in NOTCH3. This mutation was previously associated with the cerebral autosomal dominant arteriopathy with subcortical infarcts and leukoencephalopathy (CADASIL) phenotype and not associated with AD [24].

Copy Number Variants Copy number variants (CNVs) represent a final source of genetic variation that may be associated with the risk of $\mathrm{AD}$. An initial study identified variation in 
APP CNVs as underlying familial AD [25]. In a follow-up study of ten families, ten novel private CNVs segregated with EOAD (or families with mixed EOAD/LOAD onset types). The CNVs were noted to be in gene-rich areas, suggesting that these areas may be linked with AD pathogenesis [26]. However, on the whole, this type of genetic variation has not been consistently associated with AD.

\section{Conclusion: Perspective on Pathways}

The findings from large-scale GWASs, endophenotype-based GWASs, and studies that use WES and WGS collectively suggest that, apart from a few relatively rare though biologically highly informative causes of $\mathrm{AD}$, much of the variance accounting for genetic risk in $\mathrm{AD}$ occurs through common variants that individually contribute modest amounts of risk. This phenomenon has also been observed in other complex genetic diseases that are characterized by both substantial genetic contributions and environmental factors. Perhaps the most promising feature of these early days of "big data" AD genetic research is that the genetic findings discussed here are gradually illuminating the biochemical pathways underlying the pathogenesis of $\mathrm{AD}$; as more genes are identified through large-scale GWASs, WES/WGS, and novel combinations of these methodologies, a picture of the pathophysiology of $\mathrm{AD}$ is gradually emerging and coming into sharper focus. Although the total genetic risk accounted for by the markers implicated in $\mathrm{AD}$ since the discovery of $A P O E$ continue to be relatively small, the discovery of these markers has great potential for understanding the disease. Indeed, with the increasing profusion of genetic markers that can reasonably be associated with particular genes, it has become possible to begin placing these markers into potential pathways, which in turn might be targeted for novel AD treatments. And, as we have noted, while the genetic risk attributable to any given locus may be small, treatments targeting the pathways implicated by these loci may have much larger therapeutic effects.

Acknowledgments This material is based upon work supported (or supported in part) by the Office of Research and Development Medical Research R\&D Service, Department of Veterans Affairs. DWT is supported by NIA P50 AG005136, U01 AG032984, and UF1 AG047133.

The authors wish to thank Andrew David for his editorial assistance. Dr. Emil Coccaro reviewed this article.

\section{Compliance with Ethics Guidelines}

Conflict of Interest Matthew Schreiber, Debby Tsuang, and Thomas Bird have no conflicts of interest.

Human and Animal Rights and Informed Consent This article does not contain any studies with human or animal subjects performed by the authors.

\section{References}

1. Bird TD. Alzheimer disease overview. In: Pagon RA, Adam MP, Ardinger HH, Bird TD, Dolan CR, Fong C-T, et al., editors. GeneReviews [internet]. Seattle: University of Washington; 1998. http://www.ncbi.nlm.nih.gov/books/NBK1116/. Accessed 3 Apr 2014.

2. Wijsman EM, Pankratz ND, Choi Y, Rothstein JH, Faber KM, Cheng $\mathrm{R}$, et al. Genome-wide association of familial late-onset Alzheimer's disease replicates BIN1 and CLU and nominates CUGBP2 in interaction with APOE. PLoS Genet. 2011;7(2):e1001308. doi:10.1371/ journal.pgen.1001308.

3. Bettens K, Sleegers K, Van Broeckhoven C. Genetic insights in Alzheimer's disease. Lancet Neurol. 2013;12(1):92-104. doi:10. 1016/S1474-4422(12)70259-4.

4. Harold D, Abraham R, Hollingworth P, Sims R, Gerrish A, Hamshere ML, et al. Genome-wide association study identifies variants at CLU and PICALM associated with Alzheimer's disease. Nat Genet. 2009;41(10):1088-93. doi:10.1038/ng.440.

5. Seshadri S, Fitzpatrick AL, Ikram MA, DeStefano AL, Gudnason V, Boada M, et al. Genome-wide analysis of genetic loci associated with Alzheimer disease. JAMA. 2010;303(18):1832-40. doi:10.1001/ jama.2010.574.

6. Lambert JC, Heath S, Even G, Campion D, Sleegers K, Hiltunen M, et al. Genome-wide association study identifies variants at CLU and CR1 associated with Alzheimer's disease. Nat Genet. 2009;41(10): 1094-9. doi:10.1038/ng.439.

7. Naj AC, Jun G, Beecham GW, Wang LS, Vardarajan BN, Buros J, et al. Common variants at MS4A4/MS4A6E, CD2AP, CD33 and EPHA1 are associated with late-onset Alzheimer's disease. Nat Genet. 2011;43(5):436-41. doi:10.1038/ng.801.

8. Hollingworth P, Harold D, Sims R, Gerrish A, Lambert JC, Carrasquillo $\mathrm{MM}$, et al. Common variants at ABCA7, MS4A6A/MS4A4E, EPHA1, $\mathrm{CD} 33$ and CD2AP are associated with Alzheimer's disease. Nat Genet. 2011;43(5):429-35. doi:10.1038/ng.803.

9. Lambert JC, Ibrahim-Verbaas CA, Harold D, Naj AC, Sims R, Bellenguez C, et al. Meta-analysis of 74,046 individuals identifies 11 new susceptibility loci for Alzheimer's disease. Nat Genet. 2013;45(12):1452-8. doi:10.1038/ng.2802.

10. Logue MW, Schu M, Vardarajan BN, Buros J, Green RC, Go RC, et al. A comprehensive genetic association study of Alzheimer disease in African Americans. Arch Neurol. 2011;68(12):1569-79. doi: 10.1001/archneurol.2011.646.

11. Escott-Price V, Bellenguez C, Wang LS, Choi SH, Harold D, Jones L, et al. Gene-wide analysis detects two new susceptibility genes for Alzheimer's disease. PLoS One. 2014;9(6):e94661. doi:10.1371/ journal.pone.0094661.

12. Kamboh MI, Demirci FY, Wang X, Minster RL, Carrasquillo MM, Pankratz VS, et al. Genome-wide association study of Alzheimer's disease. Transl Psychiatry. 2012;2:e117. doi:10.1038/tp.2012.45.

13. Shulman JM, Chen K, Keenan BT, Chibnik LB, Fleisher A, Thiyyagura P, et al. Genetic susceptibility for Alzheimer disease neuritic plaque pathology. JAMA Neurol. 2013;70(9):1150-7. doi: 10.1001/jamaneurol.2013.2815.

14. Sherva R, Tripodis Y, Bennett DA, Chibnik LB, Crane PK, de Jager $\mathrm{PL}$, et al. Genome-wide association study of the rate of cognitive decline in Alzheimer's disease. Alzheimers Dement. 2014;10(1):4552. doi:10.1016/j.jalz.2013.01.008.

15. Cruchaga C, Kauwe JS, Harari O, Jin SC, Cai Y, Karch CM, et al. GWAS of cerebrospinal fluid tau levels identifies risk variants for Alzheimer's disease. Neuron. 2013;78(2):256-68. doi:10.1016/j. neuron.2013.02.026.

16. Chouraki V, De Bruijn RF, Chapuis J, Bis JC, Reitz C, Schraen S, et al. A genome-wide association meta-analysis of plasma $\mathrm{A} \beta$ peptides concentrations in the elderly. Mol Psychiatry. 2014. doi:10. 1038/mp.2013.185. 
17. Pottier C, Hannequin D, Coutant S, Rovelet-Lecrux A, Wallon D, Rousseau S, et al. High frequency of potentially pathogenic SORL1 mutations in autosomal dominant early-onset Alzheimer disease. Mol Psychiatry. 2012;17(9):875-9. doi:10.1038/mp.2012.15.

18. Jonsson T, Stefansson H, Steinberg S, Jonsdottir I, Jonsson PV, Snaedal J, et al. Variant of TREM2 associated with the risk of Alzheimer's disease. N Engl J Med. 2013;368(2):107-16. doi:10. 1056/NEJMoa1211103.

19. Guerreiro R, Hardy J. TREM2 and neurodegenerative disease [letter]. N Engl J Med. 2013;369(16):1569-70.

20. Bird TD. TREM2 and neurodegenerative disease [letter]. N Engl J Med. 2013;369(16):1568. doi:10.1056/NEJMc1306509\#SA5.

21. Benitez BA, Jin SC, Guerreiro R, Graham R, Lord J, Harold D, et al. Missense variant in TREML2 protects against Alzheimer's disease. Neurobiol Aging. 2014;35(6):1510.e19-26. doi:10.1016/j. neurobiolaging.2013.12.010.

22. Cruchaga C, Karch CM, Jin SC, Benitez BA, Cai Y, Guerreiro R, et al. Rare coding variants in the phospholipase D3 gene confer risk for Alzheimer's disease. Nature. 2014;505(7484):550-4. doi:10. 1038/nature12825.
23. Jonsson T, Atwal JK, Steinberg S, Snaedal J, Jonsson PV, Bjornsson $\mathrm{S}$, et al. A mutation in APP protects against Alzheimer's disease and age-related cognitive decline. Nature. 2012;488(7409):96-9. doi:10. 1038/nature11283.

24. Guerreiro RJ, Lohmann E, Kinsella E, Brás JM, Luu N, Gurunlian N, et al. Exome sequencing reveals an unexpected genetic cause of disease: NOTCH3 mutation in a Turkish family with Alzheimer's disease. Neurobiol Aging. 2012;33(5):1008.e17-23. doi:10.1016/j. neurobiolaging.2011.10.009.

25. Hooli BV, Mohapatra G, Mattheisen M, Parrado AR, Roehr JT, Shen $\mathrm{Y}$, et al. Role of common and rare APP DNA sequence variants in Alzheimer disease. Neurology. 2012;78(16):1250-7. doi:10.1212/ WNL.0b013e3182515972.

26. Hooli BV, Kovacs-Vajna ZM, Mullin K, Blumenthal MA, Mattheisen $\mathrm{M}$, Zhang $\mathrm{C}$, et al. Rare autosomal copy number variations in early-onset familial Alzheimer's disease. Mol Psychiatry. 2014;19(6):676-81. doi:10.1038/mp.2013.77.

27. Bekris LM, Yu CE, Bird TD, Tsuang DW. Genetics of Alzheimer disease. J Geriatr Psychiatry Neurol. 2010;23(4):213-27. doi:10. $1177 / 0891988710383571$. 